\title{
ISOLATION OF CD248-EXPRESSING STROMAL VASCULAR FRACTION FOR TARGETED IMPROVEMENT OF WOUND HEALING
}

Elizabeth Brett ${ }^{1}$, MS; Elizabeth R. Zielins ${ }^{1}, \mathrm{MD}$, Monica Chin ${ }^{1}$, BS, Michael Januszyk ${ }^{1}$, MD, Charles P. Blackshear ${ }^{1}$, Michael Findlay ${ }^{1}$, MD, Arash Momeni ${ }^{1}$, MD, Geoffrey C. Gurtner ${ }^{1}$, MD, Michael T. Longaker $^{1,2}$, MD, MBA, Derrick C. Wan ${ }^{1}$, MD

${ }^{1}$ Hagey Laboratory for Pediatric Regenerative Medicine, Department of Surgery, Division of Plastic Surgery, Stanford University School of Medicine, Stanford, California, USA

${ }^{2}$ Institute for Stem Cell Biology and Regenerative Medicine, Stanford University School of Medicine, Stanford, California, USA

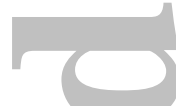

${ }^{*}$ Correspondence:

Derrick C. Wan, MD

Associate Professor of Surgery, Stanford University Medical Center

Stanford University School of Medicine

257 Campus Drive

Stanford, CA 94305

Tel 650-736-2776

Email: dwan@stanford.edu

This is the author manuscript accepted for publication and has undergone full peer review but has not been through the copyediting, typesetting, pagination and proofreading process, which may lead to differences between this version and the Version record. Please cite this article as doi:10.1111/ wrr.12542. 


\section{Financial Disclosure and Products:}

None of the authors has a financial interest in any of the products, devices, or drugs mentioned in this manuscript. M.T.L. was supported by NIH grants R21 DE024230-02, U01 HL099776, R01 DE021683, the Oak Foundation, Hagey Laboratory for Pediatric Regenerative Medicine, and the Gunn/Olivier Fund. D.C.W. was supported by NIH grant 1 K08 DE024269-01, the Hagey Laboratory for Pediatric Regenerative Medicine, and the Stanford University Child Health Research Institute Faculty Scholar Award.

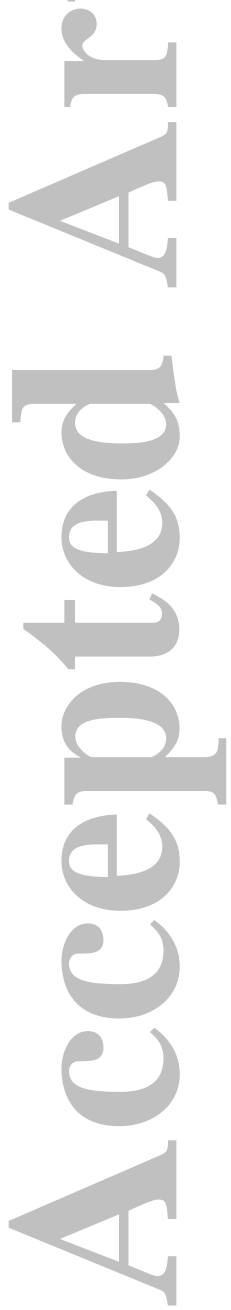




\section{ABSTRACT}

Wound healing remains a global issue of disability, cost, and health. Addition of cells from the stromal vascular fraction of adipose tissue has been shown to increase the rate of full thickness wound closure. The present study aimed to investigate the angiogenic mechanisms of CD248+ stromal vascular fraction (SVF) cells in the context of full thickness excisional wounds. Single cell transcriptional analysis was used to identify and cluster angiogenic gene-expressing cells, which was then correlated with surface marker expression. SVF cells isolated from human lipoaspirate were FACS sorted based on the presence of CD248. Cells were analyzed for angiogenic gene expression and ability to promote microvascular tubule formation in vitro. Following this, $6 \mathrm{~mm}$ full thickness dermal wounds were created on the dorsa of immunocompromised mice and then treated with CD248+, CD248-, or unsorted SVF cells delivered in a pullalan-collagen hydrogel or the hydrogel alone. Wounds were measured every other day photometrically until closure. Wounds were also evaluated histologically at 7 and 14 days post-wounding and when fully healed to assess for re-epithelialization and development of neovasculature. Wounds treated with CD248+ cells healed significantly faster than other treatment groups, and at 7 days, had quantitatively more re-epithelialization. Concurrently, immunohistochemistry of CD31 revealed a much higher presence of vascularity in the CD248+ SVF cells treated group at the time of healing and at 14 days post-op, consistent with a proangiogenic effect of CD248+ cells in vivo. Therefore, using CD248+ pro-angiogenic cells obtained from SVF presents a viable strategy in wound healing by promoting increased vessel growth in the wound.

Keywords: angiogenesis, CD248, full thickness dermal wounds, wound healing 


\section{Introduction}

Cells within the stromal vascular fraction (SVF) have been extensively studied in the context of promoting diabetic wound healing (1), enhancing fat graft retention in irradiated and non-irradiated settings $(2,3)$, and for reconstruction of calvarial defects (4). However, the exact mechanisms of action of these transplanted cells in regenerating tissue/restoring damage are not fully known. It can be postulated that the stemness of this population leads to differentiation into the target tissue through influence of the local microenvironment. It is also known, though, that implanted cells can deliver growth factors in a paracrine fashion $(5,6)$. Secreted factors by SVF cells have been shown to induce tubule formation in vitro by human microvascular endothelial cells (7).

Importantly, the heterogeneity of SVF cells has limited the ability for precise, unambiguous delineation of cellular function, particularly with bulk population transcriptional analyses. This heterogeneity also poses a significant challenge for use of these cells in translational applications. Subpopulations within SVF cells have been documented with disparate biologic activity and potentials for lineage differentiation, and this carries important therapeutic implications for clinical use where efficacy and reproducibility is key. Recent development of single-cell transcriptional assays has begun to yield meaningful information regarding biologic function of individual cells, and this has guided selection of novel subpopulations for various purposes (8-11).

Microfluidic single cell analysis allows for evaluation of transcriptional profiles of multiple individual cells, which can facilitate identification and isolation of pro-angiogenic subpopulations using flow cytometry. Microfluidic analysis of cells within the SVF has already proven useful in identifying cell surface markers indicating pro-osteogenic cell populations. Through this approach, subpopulations isolated based on CD105, CD90, or BMPR-IB expression have all been shown to 
enhance bone regeneration in an in vivo mouse calvarial defect model $(9,12,13)$. It is thus possible to interrogate a heterogeneous cell population and cluster the transcriptional data output based on specific gene expression (14), and organization of cell phenotypes by proxy of expressed genes can allow for recognition and isolation of desired subpopulations within a larger heterogeneous mix.

1

In this present study, a bioinformatics approach to examine pro-angiogenic cells via gene expression profiles (VEGF, FGF2, PDGFRa, and PDGFR $\beta$ ) was employed, and we identified CD248 as a significantly expressed surface marker among cells with high levels of angiogenic gene transcripts. We then investigated the in vitro gene expression profile of CD248+ cells and their ability to promote tubule formation by human microvascular endothelial cells. Having determined the efficacy of this population in vitro, we finally investigated the potential of CD248+ cells to contribute to faster wound healing in vivo.

\section{Materials and Methods}

Human Adipose-Derived Stromal Vascular Fraction Harvest

Human lipoaspirate specimens from the abdomen were obtained from five healthy female donors following informed consent under Stanford Institutional Review Board approval (Protocol no. 2188). Donors were between the ages of $35-50$ and had no medical comorbidities. The lipoaspirate was processed to obtain SVF cells. Briefly, the lipoaspirate was washed twice with phosphate-buffered saline (PBS) and then digested using type II collagenase (Sigma-Aldrich; St. Louis, MO) in Medium 199 (Cellgro; Manassas, VA) at $37^{\circ} \mathrm{C}$ under gentle agitation for 30 minutes. Digestion was neutralized with an equal volume of Dulbecco's Modified Eagle's Medium plus GlutaMAX (DMEM; Invitrogen; Carlsbad, CA) supplemented with $10 \%$ fetal bovine serum (FBS) 
and centrifuged at $1500 \mathrm{rpm}$ for 20 minutes. The supernatant was aspirated and the cell pellet was resuspended in DMEM and then passed through a $100 \mu \mathrm{m}$ pore cell strainer (Corning; Corning, NY) before being re-centrifuged at $1500 \mathrm{rpm}$ for 15 minutes. The cell pellet was resuspended in red cell lysis buffer and centrifuged again at $1500 \mathrm{rpm}$ for 15 minutes, leaving a stromal vascular fraction pellet. The number of live SVF cells were counted using trypan blue.

\section{Microfluidic Chip-Based Single Cell Analysis}

Microfluidic Chip-Based Single Cell transcriptional analysis was performed as previously described $(11,15-18)$. Single cells were sorted into each well of a 96-well plate, which was preloaded with $10 \mu \mathrm{L}$ of a master mix containing Tris-EDTA buffer (pH 7.0), Superscript III reverse transcriptase enzyme (Invitrogen, Carlsbad, CA), Cells Direct reaction mix (Invitrogen, Carlsbad, CA), target gene-specific TaqMan assay (primer/probe) sets (Applied Biosystems, Foster City, CA), and SUPERase-In RNAse inhibitor (Applied Biosystems, Foster City, CA). Exon-spanning primers were selected to avoid amplification of genomic background. Cells were then lysed and reverse transcription was performed (20 minutes at 50C, 2 minutes at 95C), followed by genespecific amplification of the cDNA (22-cycles, 95C for 15 minutes then $60 \mathrm{C}$ for 4 minutes). Resultant amplified single cell cDNA was then mixed with sample loading agent (Fluidigm, South San Francisco, CA) and Universal PCR Master Mix (Applied Biosystems, Foster City, CA) and placed into individual wells of 48.48 Dynamic Array chips (Fluidigm, South San Francisco, CA). TaqMan probes and assay loading agent were also placed into individual wells according to the manufacturer's instructions (Fluidigm, South San Francisco, CA). Products were analyzed on the BioMark reader system (Fluidigm, South San Francisco, CA) using a hot start protocol, and 30 quantitative PCR cycles were performed (18). 


\section{Surface Marker Correlation}

Expression data from microfluidic chips were normalized relative to median expression for each gene and converted to base 2 logarithms. A Fuzzy C-Means clustering algorithm was employed to cluster groups of cells based on similarities in gene expression $(9,18)$. Cluster designations were then flattened to assign each cell the single cluster for which it associated the highest membership, thus creating two mutually exclusive sets. Linear discriminant analysis was applied to determine genes whose expression patterns maximally differed between the two groups and a receiver operating characteristic analysis was used to create a feature subset of genes with corresponding partition function (VEGF, FGF2, PDGFRA, and PDGFRB). Finally, linear discriminant analysis was employed to identify surface marker genes whose expression was best able to distinguish between the two groups $(9,18)$.

\section{FACS Isolation of CD248 Cell Populations}

Freshly harvested SVF cells from lipoaspirate were resuspended in FACS buffer (1XPBS, 2\% fetal bovine serum, 1\% P188, 1\% Pen-Strep, Gibco), and were stained with anti-CD248 antibody (human anti-CD248 primary antibody, ab115870 at 1:100, Abcam, Cambridge, UK). Flow cytometry was used in order to quantify the proportion of SVF cells which express CD248. Positive and negative populations were then sorted using a FACS Aria II (BD Biosciences, San Jose, CA) with a $100 \mu \mathrm{m}$ nozzle.

\section{Angiogenic Gene Expression}

RNA for gene expression assay was collected at day 0, from fresh SVF cells, harvested using TRIzol (Life Technologies) and processed using the RNeasy Mini Kit (Qiagen; Hilden, Germany). Reverse transcription was performed using TaqMan Reverse Transcription Reagents 
(Invitrogen). An ABI Prism 7900HT Sequence Detection System (Applied Biosystems; Foster City, CA) was used to perform quantitative real-time polymerase chain reaction (qRT-PCR) with Power SYBR Green PCR Master Mix (Applied Biosystems) as the reporter. qRT-PCR analysis was conducted to detect gene expression levels of pro-angiogenic hepatocyte growth factor (HGF) and vascular endothelial growth factor (VEGF). Expression levels of all genes were normalized to GAPDH expression values.

In Vitro Endothelial Tube Formation Assay

To further explore the proangiogenic effect of CD248+ SVF cells, an endothelial tubule formation assay was performed. Human microvascular endothelial cells were seeded at a density of $1.2 \times 10^{5}$ cells per well on Geltrex-coated 24-well plates in unsupplemented endothelial cell media (Thermo Fisher, Waltham MA). FACS-sorted CD248+ and CD248-cell populations and unsorted SVF cells were seeded in cell culture inserts at a density of $7.5 \times 10^{3}$ cells per insert. As a control, endothelial cells were also treated with $10 \mathrm{ng} / \mathrm{ml}$ recombinant VEGF alone. Endothelial cells were incubated for 18 hours and stained with Calcein AM immediately before imaging. Tubule analysis was performed with the Angiogenesis Analyzer tool for ImageJ which measures the total area of tube formation/mesh area, master segments which consist of pieces of the network delimited by two junctions, and master junctions which are intersections linking at least three master segments in each field of view.

\section{In Vivo Full Thickness Dorsal Wounds}

All experiments were performed in accordance with the Stanford University Animal Care and Use Committee Guidelines and approved APLAC protocols. Two $6 \mathrm{~mm}$ full thickness wounds were made at the same level on the dorsum of CD1 athymic nude mice, as previously described 
(19). A silicone $12 \mathrm{~mm}$ diameter washer (Invitrogen) was placed around the perimeter of the wound and secured with cyanoacrylate glue and interrupted Ethilon 6-0 sutures (eSutures.com, Mokena, IL) to prevent wound contracture. Mice were randomized into four groups ( $n=11$ animals per group, thus 22 wounds per group) receiving different treatments including: pullalan-collagen hydrogel alone, unsorted SVF cells, CD248-SVF cells, or CD248+ SVF cells in a pullalan-collagen hydrogel. A total of 250,000 cells were added to each wound with $20 \mu$ l of PBS. The hydrogel alone received $20 \mu \mathrm{l}$ of PBS as a vehicle control. Wounds were dressed with Tegaderm sterile dressing (3M Healthcare, St Paul, MN), which was changed post-op day 3 , followed by every other day until wound closure. For each treatment group, mice were sacrificed in 3 different time points; 3 mice at day 7, 3 mice at day 14 , and 5 mice at the day of closure for each specific group. Digital photographs were taken at the time of surgery and every dressing change until closure, defined as the time at which the wound bed was completely re-epithelialized and filled with new tissue. Wound area was quantified using ImageJ software (NIH, Bethesda, MD) and expressed as a ratio of wound circumference to silicone stent circumference.

Histological Analysis of Wounds

Mice were sacrificed and dorsal skin was harvested for histological analysis at either day 7,14 , or at time of wound healing. Briefly, skin was fixed in $4 \%$ paraformaldehyde and subsequently dehydrated and embedded for paraffin sectioning. Sections were cut at $8 \propto \mathrm{m}$ and immunohistochemical staining was used at 7 days to assess levels of VEGF and CD248 in the non-healed wounds (anti-mouse VEGF, ab46154 at 1:50, and CD248 primary antibody, ab115870 at 1:50, Abcam). Tissue harvested on day 7 was also sectioned, H\&E stained, and epithelialization was assessed from slides at the center of the wound as a percentage of the original wound ( $n=10$ sections per wound). At day 14, and at wound closure per group, CD31 
staining was performed to assess vasculature (anti-mouse CD31 primary antibody, ab28364 at 1:50, Abcam), and CD248 and VEGF stains were also performed to assess early pro-angiogenic changes in the extracellular matrix.

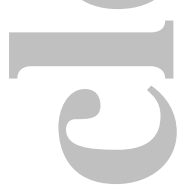

\section{Statistical Analyses}

Data are presented as means \pm standard deviation (SD). Analyses of variance (ANOVA) were used for multiple group comparisons. A * $p$-value of $<0.05$ was considered to be statistically significant. All statistical analyses were performed using GraphPad Prism software (GraphPad Software Inc.; La Jolla, CA). For single-cell transcriptional data, a Kolmogorov-Smirnov test was used to compare empirical distributions, followed by an adaptive fuzzy c-means clustering algorithm as described above (18).

\section{Results}

CD248 is expressed by cells which secrete VEGF

Within the heterogeneous population of SVF cells in lipoaspirate, microfluidic single cells expression analysis identified a subpopulation of cells with increased expression of angiogenic genes. Using a non-parametric Kolmogorov-Smirnov test and receiver operating characteristic analysis, SVF cells could be assigned membership into one of two clusters based on expression of multiple angiogenic genes (VEGF, FGF2, PDGFRA, and PDGFRB) (Figure 1A). Linear discriminant analysis was then performed to correlate cell surface marker expression with the angiogenic SVF cell population in question, and a significant correlation between CD248 expression and clustering of SVF cells based on angiogenic gene expression was noted (Figure 1B) $\left({ }^{*} p<0.05\right)$. FACS analysis revealed that SVF was $14.8 \%$ positive for CD248 (Figure 1C). 
CD248+ cells express significantly higher pro-angiogenic genes, and induce higher quantities of robust tubules in vitro

Gene expression analysis was performed for HGF and VEGF on CD248+/- and unsorted cells. CD248+ cells were found to express significantly elevated levels of HGF and VEGF in comparison to CD248- SVF cells and unsorted SVF cells $\left({ }^{*} p<0.05,{ }^{* *} p<0.01\right)$ (Figure 2A). CD248+ SVF cells also enhanced the ability of human microvascular endothelial cells to form tubules in vitro. Specifically, CD248+ SVF cells induced a statistically significant increase in the total area of tube formation (percent mesh area), number of nodes/master junctions, and number of tubules/segments formed by microvascular endothelial cells when compared to CD248- and unsorted SVF cells $\left({ }^{*} p<0.05\right)$ (Figure 2B and C). Furthermore, the effects of CD248+ SVF cells on endothelial cells were similar or greater than that observed with exogenous VEGF control.

CD248+ cells lead to faster healing of wounds with more vascularity

To evaluate the ability of SVF cell subpopulations to enhance wound healing, bilateral full thickness excisional wounds were created on the dorsa of immunocompromised mice. Each wound was then supplied with a pullalan-collagen hydrogel, and subsequently treated with either CD248+ cells, CD248- cells, unsorted cells, or no cells (hydrogel alone). By 13 days postwounding, animals which received CD248+ cells healed completely, in contrast to complete healing noted at day 15 for CD248- and unsorted cell groups, and 16 days for the group which did not receive cells (Figure 3A). Using Image $\mathrm{J}$ analysis for wound area, it was seen that the group which received CD248+ cells had significantly more healing than all other groups (CD248, unsorted cells, and hydrogel alone) by day 7, a pattern which continued through day 9 and day $11\left({ }^{*} p<0.05\right.$ for CD248+ vs. all other groups at all three time points) (Figure 3B). Wounds 
harvested at day 7 demonstrated greater VEGF and CD248 staining in the CD248+ group when compared to the CD248-, unsorted, and hydrogel alone groups (Figure 4A), and these differences were found to be significant (Figure 4B) $\left({ }^{*} p<0.05\right)$. While CD31 staining at 7 days post-op showed no significant differences (Figure 5A and bottom of Figure 5C), H\&E staining of the entire wound showed keratinocyte migration over the wounded dermis to be greatest when treated with CD248+ cells compared to the other three groups (Figure 5B and top of Figure 5C). Wounds were also analyzed at 14 days post-op across all groups (Figure 6A). Significantly more CD31 staining was found between the wounds treated with CD248+ cells when compared to the remaining three groups (Figure $6 \mathrm{~B})\left({ }^{*} p<0.05\right)$. Once wounds were fully healed, the remaining mice were sacrificed and wounds were harvested for immunohistochemistry analysis and H\&E staining (Figure 6C). At this later timepoint, anti-CD31 staining again revealed significantly higher percentage of positive staining in the CD248+ group when compared to the remaining groups (Figure 6D) $\left({ }^{*} p<0.05\right)$.

\section{Discussion}

CD248, also known as endosialin and tumor endothelial marker 1 (TEM-1), does not have a fully characterized role, which is reflected in some conflicting literature. While some describe CD248 as a marker of tumor related myofibroblasts (20), it has also been described as a marker of stromal fibroblasts, which is not exclusively expressed on tumor endothelium (21). Furthermore, endosialin expression has also been noted in pericytes, and studies have shown CD248 is necessary for capillary sprouting in skeletal muscle angiogenesis (22). Endosialin may thus influence angiogenic activity in vivo.

The findings presented here demonstrate the potential of CD248 as a marker of a proangiogenic subpopulation of SVF cells, and increased expression of HGF and VEGF among 
CD248+ SVF cells may contribute to observed enhanced wound healing potential of these cells. VEGF is known to assist wound healing by promoting endothelial cell migration and proliferation (23), and HGF has also been shown to be an important cytokine for angiogenesis (24). Furthermore, HGF functions as a hepatotrophic and renotrophic agent for liver and kidney regeneration (25), and HGF is secreted by dermal fibroblasts as a $90 \mathrm{kDa}$ monomer, which becomes active when proteolytically converted to a heterodimer (26). Upon wounding, HGF is released from dermal fibroblasts and induces a rapid morphology change in overlying keratinocytes. The change from round, compact cells bound by desmosomal junctions to unbound cells allow them to rapidly cover more surface area, hence helping their role in fast restoration of the integrity of the epithelial barrier (27). Consistent with this, at our early in vivo time point of 7 days, we observed quantitatively increased migration of keratinocytes in the CD248+ SVF cell treated group. Also observed at 7 days was an increase in VEGF staining in the extracellular matrix of CD248+ SVF cell treated group, which was subsequently associated with increased CD31 staining at the time of wound closure.

The angiogenic effect of CD248+ cells has been demonstrated here in vitro and in vivo. While it is possible that CD248+ SVF cells, suggested by some studies to contain pericytes (28), may contribute directly to vessel formation themselves, it is also important to note the profound effect their paracrine presence had on endothelial cells within in vitro culture, with a similar or greater pro-angiogenic effect observed than in our exogenous VEGF positive control. The impact of factors secreted by CD248+ cells (both VEGF and HGF) on the overlying endothelial cell culture helps to explain the significant vascularization the corresponding wound healing exhibited in vivo (7). Importantly, continued expression of these factors may also contribute to reduced vessel regression during late phases of wound healing, as greater CD31 staining was still appreciated among CD248+ SVF cell treated wounds compared to other groups after healing was noted at day 14 . 
While it is acknowledged that VEGF acts as a mitogen for endothelial cells through the mechanism of mitogen activated protein kinases (MAPKs) (29), the governance of CD248 expression and how this may relate to angiogenic gene expression remains undefined. Studies have shown that CD248 is associated with developing vasculature (30), and there is also a link between the expression of CD248 and HIF-2a, a mediator of the hypoxia pathway. CD248 gene expression is driven by a hypoxic response element promoter, leading to its activation in low oxygen pressure environments (31). CD248 has also been associated with rapid neovascularization in developing fetal tissue (30). Therefore, the isolation and use of a CD248+ population of cells may enhance angiogenesis within transplanted tissue and hypoxic wound environments.

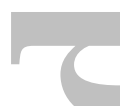

Importantly, while considering the pro-angiogenic, pro-healing impact that CD248+ SVF cells may possess, it is also critical to consider the actual fraction of SVF which is CD248+, as FACS analysis revealed this to be approximately $15 \%$. As SVF cells are known to be remarkably heterogeneous, it is therefore not surprising that unsorted SVF cells provided minimal enhancement of wound healing in our study, as the myriad cell populations comprising the remaining $85 \%$ may be neutral or even detrimental to re-epithelialization.

Nonetheless, the isolation of a CD248+ subpopulation of SVF cells and use thereof in a wounding model in vivo highlights the ability of these cells to form vasculature and otherwise rescue an area of hypoxic, healing tissue. Therefore, isolating pro-angiogenic cells using this marker may have many potential promising applications where revascularization is necessary. Conversely, studying CD248 also stands to answer some important questions regarding neovascularization (32), and perhaps may be a marker which can be targeted for tumor treatment. 
References

1. Kuo YR, Wang CT, Cheng JT, Kao GS, Chiang YC, Wang CJ. Adipose-Derived Stem Cells Accelerate Diabetic Wound Healing Through the Induction of Autocrine and Paracrine Effects. Cell transplantation. 2016;25(1):71-81.

2. Luan A, Duscher D, Whittam AJ, Paik KJ, Zielins ER, Brett EA, et al. Cell-Assisted Lipotransfer Improves Volume Retention in Irradiated Recipient Sites and Rescues Radiation-Induced Skin Changes. Stem cells (Dayton, Ohio). 2016;34(3):668-73.

3. Garza RM, Rennert RC, Paik KJ, Atashroo D, Chung MT, Duscher D, et al. Studies in fat grafting: Part IV. Adipose-derived stromal cell gene expression in cell-assisted lipotransfer. Plastic and reconstructive surgery. 2015;135(4):1045-55.

4. Levi B, James AW, Nelson ER, Vistnes D, Wu B, Lee M, et al. Human Adipose Derived Stromal Cells Heal Critical Size Mouse Calvarial Defects. PLoS ONE. 2010;5(6).

5. Hsiao ST, Lokmic Z, Peshavariya H, Abberton KM, Dusting GJ, Lim SY, et al. Hypoxic conditioning enhances the angiogenic paracrine activity of human adipose-derived stem cells. Stem cells and development. 2013;22(10):1614-23.

6. Baraniak PR, McDevitt TC. Stem cell paracrine actions and tissue regeneration. Regenerative medicine. 2010;5(1):121-43.

7. Lopatina T, Kalinina N, Karagyaur M, Stambolsky D, Rubina K, Revischin A, et al. Adipose-derived stem cells stimulate regeneration of peripheral nerves: BDNF secreted by these cells promotes nerve healing and axon growth de novo. PLoS ONE. 2011;6.

8. Lauvrud AT, Kelk P, Wiberg M, Kingham PJ. Characterization of human adipose tissue-derived stem cells with enhanced angiogenic and adipogenic properties. Journal of tissue engineering and regenerative medicine. 2016.

9. Levi B, Wan DC, Glotzbach JP, Hyun J, Januszyk M, Montoro D, et al. CD105 protein depletion enhances human adipose-derived stromal cell osteogenesis through reduction of transforming growth factor beta1 (TGF-beta1) signaling. The Journal of biological chemistry. 2011;286(45):39497-509.

10. Zielins ER, Paik K, Ransom RC, Brett EA, Blackshear CP, Luan A, et al. Enrichment of AdiposeDerived Stromal Cells for BMPR1A Facilitates Enhanced Adipogenesis. Tissue engineering Part A. 2016;22(3-4):214-21.

11. Rennert RC, Januszyk M, Sorkin M, Rodrigues M, Maan ZN, Duscher D, et al. Microfluidic singlecell transcriptional analysis rationally identifies novel surface marker profiles to enhance cell-based therapies. Nature communications. 2016;7:11945.

12. McArdle A, Chung MT, Paik KJ, Duldulao C, Chan C, Rennert R, et al. Positive Selection for Bone Morphogenetic Protein Receptor Type-IB Promotes Differentiation and Specification of Human AdiposeDerived Stromal Cells Toward an Osteogenic Lineage. Tissue Eng Part A. 2014;20(21-22):3031-40.

13. Chung MT, Liu C, Hyun JS, Lo DD, Montoro DT, Hasegawa M, et al. CD90 (Thy-1)-positive selection enhances osteogenic capacity of human adipose-derived stromal cells. Tissue engineering Part A. 2013;19(7-8):989-97.

14. Reece A, Xia B, Jiang Z, Noren B, McBride R, Oakey J. Microfluidic techniques for high throughput single cell analysis. Current Opinion in Biotechnology. 2016;40:90-6.

15. Guo G, Huss M, Tong GQ, Wang C, Li Sun L, Clarke ND, et al. Resolution of cell fate decisions revealed by single-cell gene expression analysis from zygote to blastocyst. Developmental cell. 2010;18(4):675-85. 
16. Narsinh KH, Sun N, Sanchez-Freire V, Lee AS, Almeida P, Hu S, et al. Single cell transcriptional profiling reveals heterogeneity of human induced pluripotent stem cells. The Journal of clinical investigation. 2011;121(3):1217-21.

17. Diehn M, Cho RW, Lobo NA, Kalisky T, Dorie MJ, Kulp AN, et al. Association of reactive oxygen species levels and radioresistance in cancer stem cells. Nature. 2009;458(7239):780-3.

18. Glotzbach JP, Januszyk M, Vial IN, Wong VW, Gelbard A, Kalisky T, et al. An Information Theoretic, Microfluidic-Based Single Cell Analysis Permits Identification of Subpopulations among Putatively Homogeneous Stem Cells. PloS one. 2011;6(6):e21211.

19. Galiano RD, Michaels Jt, Dobryansky M, Levine JP, Gurtner GC. Quantitative and reproducible murine model of excisional wound healing. Wound repair and regeneration : official publication of the Wound Healing Society [and] the European Tissue Repair Society. 2004;12(4):485-92.

20. Christian S, Winkler R, Helfrich I, Boos AM, Besemfelder E, Schadendorf $D$, et al. Endosialin (Tem1) Is a Marker of Tumor-Associated Myofibroblasts and Tumor Vessel-Associated Mural Cells. Am J Pathol. 2008;172(2):486-94.

21. MacFadyen JR, Haworth O, Roberston D, Hardie D, Webster M-T, Morris HR, et al. Endosialin (TEM1, CD248) is a marker of stromal fibroblasts and is not selectively expressed on tumour endothelium. FEBS Letters. 2005;579(12):2569-75.

22. Naylor AJ, McGettrick HM, Maynard WD, May P, Barone F, Croft AP, et al. A differential role for CD248 (Endosialin) in PDGF-mediated skeletal muscle angiogenesis. PloS one. 2014;9(9):e107146.

23. Johnson KE, Wilgus TA. Vascular Endothelial Growth Factor and Angiogenesis in the Regulation of Cutaneous Wound Repair. Advances in Wound Care. 2014;3(10):647-61.

24. Zhao L, Liu X, Zhang Y, Liang X, Ding Y, Xu Y, et al. Enhanced cell survival and paracrine effects of mesenchymal stem cells overexpressing hepatocyte growth factor promote cardioprotection in myocardial infarction. Experimental cell research. 2016;344(1):30-9.

25. Matsumoto K, Okazaki H, Nakamura T. Up-regulation of hepatocyte growth factor gene expression by interleukin-1 in human skin fibroblasts. Biochemical and Biophysical Research Communications. 1992;188(1):235-43.

26. Dunsmore SE, Rubin JS, Kovacs SO, Chedid M, Parks WC, Welgus HG. Mechanisms of hepatocyte growth factor stimulation of keratinocyte metalloproteinase production. J Biol Chem. 1996;271(40):24576-82.

27. Seeger MA, Paller AS. The Roles of Growth Factors in Keratinocyte Migration. Advances in Wound Care. 2015;4(4):213-24.

28. Tomkowicz B, Rybinski K, Sebeck D, Sass P, Nicolaides NC, Grasso L, et al. Endosialin/TEM$1 / C D 248$ regulates pericyte proliferation through PDGF receptor signaling. Cancer biology \& therapy. 2010;9(11):908-15.

29. D'Angelo G, Struman I, Martial J, Weiner RI. Activation of mitogen-activated protein kinases by vascular endothelial growth factor and basic fibroblast growth factor in capillary endothelial cells is inhibited by the antiangiogenic factor $16-\mathrm{kDa} \mathrm{N}$-terminal fragment of prolactin. Proceedings of the National Academy of Sciences of the United States of America. 1995;92(14):6374-8.

30. Bagley RG, Honma N, Weber W, Boutin P, Rouleau C, Shankara S, et al. Endosialin/TEM 1/CD248 is a pericyte marker of embryonic and tumor neovascularization. Microvascular research. 2008;76(3):180-8.

31. Ohradanova A, Gradin K, Barathova M, Zatovicova M, Holotnakova T, Kopacek J, et al. Hypoxia upregulates expression of human endosialin gene via hypoxia-inducible factor 2 . Br J Cancer. 2008;99(8):1348-56.

32. Carson-Walter EB, Winans BN, Whiteman MC, Liu Y, Jarvela S, Haapasalo H, et al.

Characterization of TEM1/endosialin in human and murine brain tumors. BMC Cancer. 2009;9:417. 


\section{Figure Legends}

Figure 1: $(A)$ Heat maps obtained from single cell transcriptional analysis show clustering based on pro-angiogenic genes (VEGF, FGF2, PDGFRA, and PDGFRB). (B) Linear discriminant analysis revealed CD248 as the marker whose expression most significantly correlated with cluster identification. (C) Flow cytometry plot shows prevalence of CD248 positive cells obtained from SVF (79.1\% negative, $14.8 \%$ positive).

Figure 2: (A) qRT-PCR results of HGF and VEGF reveal a significant upregulation of both genes in the CD248+ populations when compared to CD248- and unsorted groups. $\left({ }^{*} p<0.05,{ }^{* *} p<\right.$ 0.01). (B) Micrographs show results from endothelial tubule formation assay, with exogenous VEGF $10 \mathrm{ng} / \mathrm{ml}$ alone serving as a positive control. Top row shows tubules stained with calcein AM, bottom row shows the computed quantities of vessel formation. (C) Graphs show quantification of the stained tubules. CD248+ cells show highest percent mesh area $\left({ }^{\star} p<0.05\right)$, and highest number of master junctions and segments $\left({ }^{*} p<0.05\right)$.

Figure 3: (A) Representative images show photometric analysis of full thickness excisional wounds across 4 groups (CD248+, CD248-, unsorted, and hydrogel alone). Black boxes indicate wound closure. (B) Quantification of wound area was performed using Image J. Graph represents numeric value of wound area across the 4 test groups from day 0 until time of wound closure. CD248+ cells are seen to accelerate wound healing significantly compared to CD248-, unsorted cells, and hydrogel alone on days 7, 9, and 11. CD248+ treated wounds healed by day 13, CD248and unsorted cell-treated wounds healed by day 15 , and hydrogel alone-treated wounds healed by day 16 ( ${ }^{*} p<0.05$ for CD248+ vs. all other groups at days 7,9 , and 11$)$.

Figure 4: A) Immunohistochemical stains of wounds for VEGF (red), CD248 (green), and DAPI (blue), with merged image on right. Scale bar $=100 \mu \mathrm{m}$. (B) Quantification of immunofluorescent images $\left({ }^{*} p<0.05\right)$.

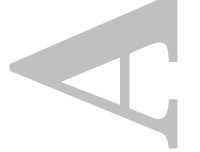

Figure 5: (A) CD31 immunohistochemical staining of wounds excised 7 days post-op. Scale bar $=100 \mu \mathrm{m}$. (B) H\&E images of full wounds. Dotted lines represent wound borders, and solid perpendicular lines highlight length of epithelial cover. Re-epithelialization is expressed as a percentage of the distance between the dotted lines. (C) Quantification of re-epithelialization (top), and quantification of CD31 staining (bottom). $\left({ }^{*} p<0.05\right)$. 
Figure 6: (A) Haematoxylin and eosin staining of excised wounds at day 14 (top row). Scale bar $=80 \mu \mathrm{m}$. Anti-CD31 (red) immunofluorescent staining (bottom row) Scale bar $=100 \mu \mathrm{m}$.

(B) Quantification of CD31 immunofluorescent images shown in 6A revealed significantly more staining in CD248+ treated wounds compared to other groups $\left({ }^{*} p<0.05\right)$. (C) H\&E staining at completion of wound healing (top row). Scale bar $=80 \mu \mathrm{m}$. Anti-CD31 (red) immunofluorescent staining (bottom row). Scale bar $=100 \mu \mathrm{m}$. (D) Quantification of CD31 immunofluorescent images shown in 6C demonstrated more nascent vessels in the wounds of CD248+ group when compared to the other 3 test groups. $\left({ }^{*} p<0.05\right)$.

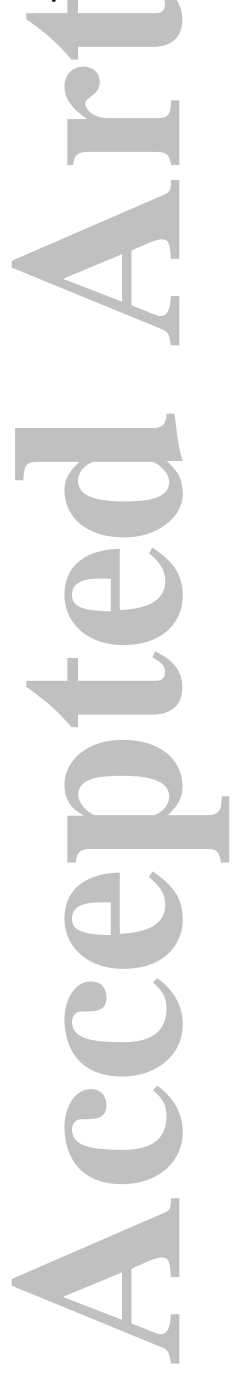


Figure 1

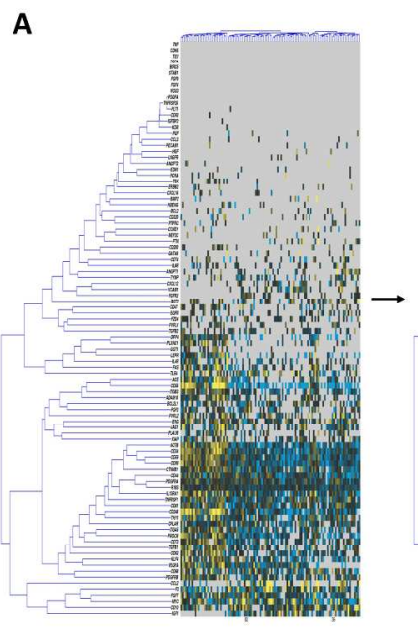



B

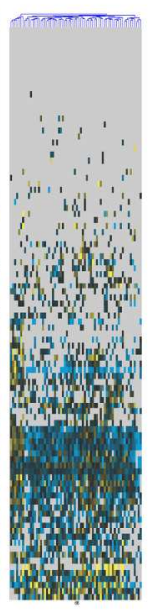

C

\begin{tabular}{|l|l|}
\hline Gene & $p$-value \\
\hline 1. CD248 & $6.81 \mathrm{e}-06$ \\
\hline 2. CD55 & $6.63 \mathrm{e}-06$ \\
\hline 3. DPP4 & $1.71 \mathrm{e}-04$ \\
\hline 4. CD99 & $1.04 \mathrm{e}-03$ \\
\hline 5. ITGB3 & $5.07 \mathrm{e}-03$ \\
\hline
\end{tabular}

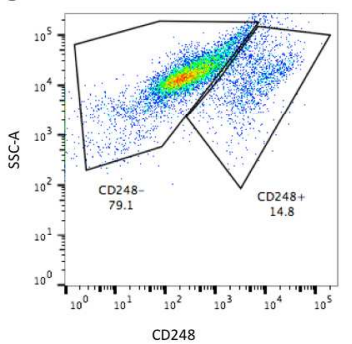

Figure 1: (A) Heat maps obtained from single cell transcriptional analysis show clustering!! + based on proangiogenic genes (VEGF, FGF2, PDGFRA, and PDGFRB). (B) Linear!! + discriminant analysis revealed CD248 as the marker whose expression most significantly! + correlated with cluster identification. (C) Flow cytometry plot shows prevalence of CD248!! + positive cells obtained from SVF $(79.1 \%$ negative, $14.8 \%$

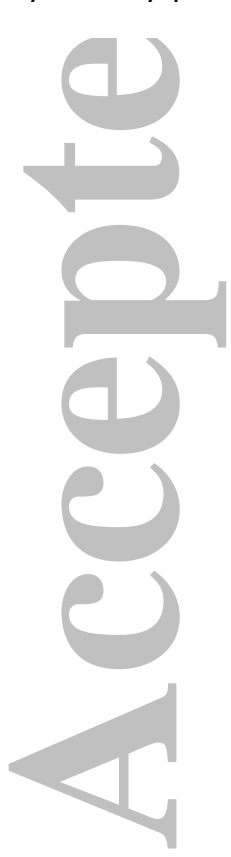
positive). 
Figure 2

A
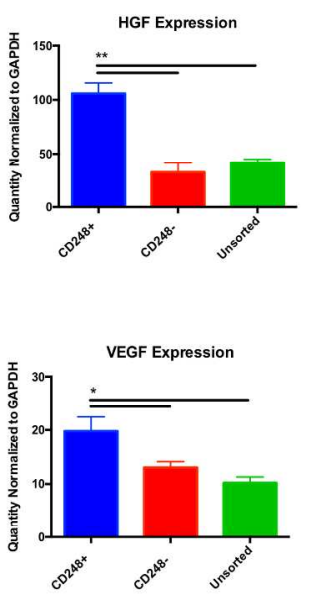

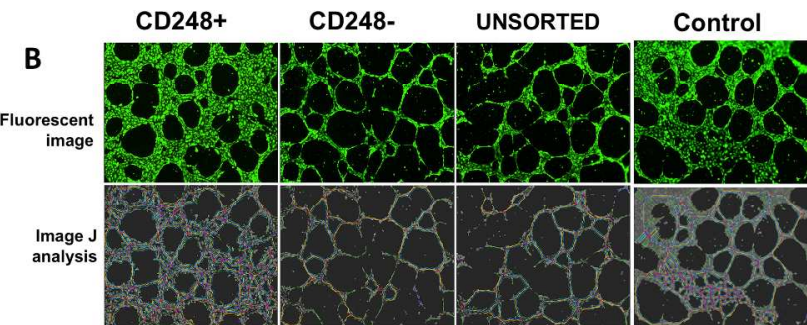

C

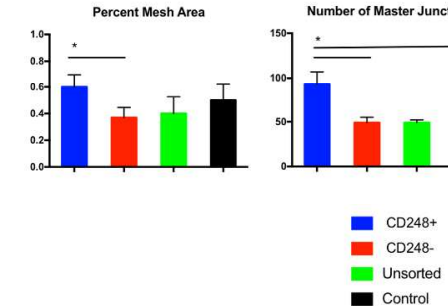

Figure 2: (A) qRT-PCR results of HGF and VEGF reveal a significant upregulation of both!! + genes in the CD248+ populations when compared to CD248- and unsorted groups. (*p <! + 0.05, **p < 0.01). (B) Micrographs show results from endothelial tubule formation assay, with!! + exogenous VEGF 10ng/ml alone serving as a positive control. Top row shows tubules!! + stained with calcein AM, bottom row shows the computed quantities of vessel formation. (C)! + Graphs show quantification of the stained tubules. CD248+ cells show highest percent mesh!! + area (*p $<0.05)$, and highest number of master junctions and

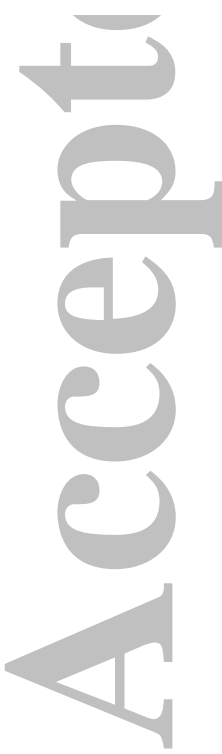
segments $(* p<0.05)$. 


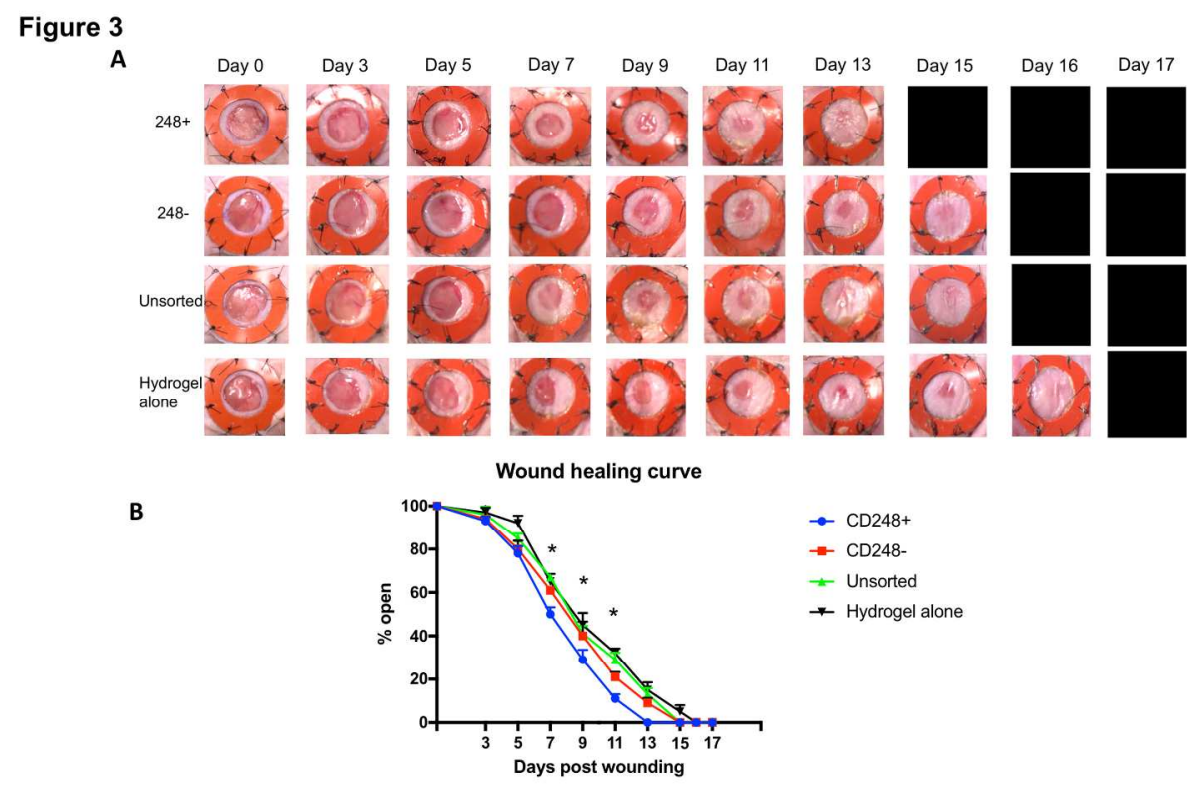

Figure 3: (A) Representative images show photometric analysis of full thickness excisional!! + wounds across 4 groups (CD248+, CD248-, unsorted, and hydrogel alone). Black boxes!! + indicate wound closure. (B) Quantification of wound area was performed using Image J.!! + Graph represents numeric value of wound area across the 4 test groups from day 0 until!! + time of wound closure. CD248+ cells are seen to accelerate wound healing significantly! + compared to CD248-, unsorted cells, and hydrogel alone on days 7,9 , and 11. CD248+!! + treated wounds healed by day 13, CD248- and unsorted cell-treated wounds healed by day! +15 , and hydrogel alone-treated wounds healed by day 16 ( $* p<0.05$ for CD248+ vs. all other!! + groups at days 7, 9, and 11).

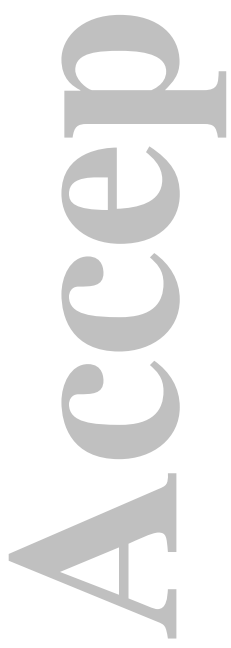




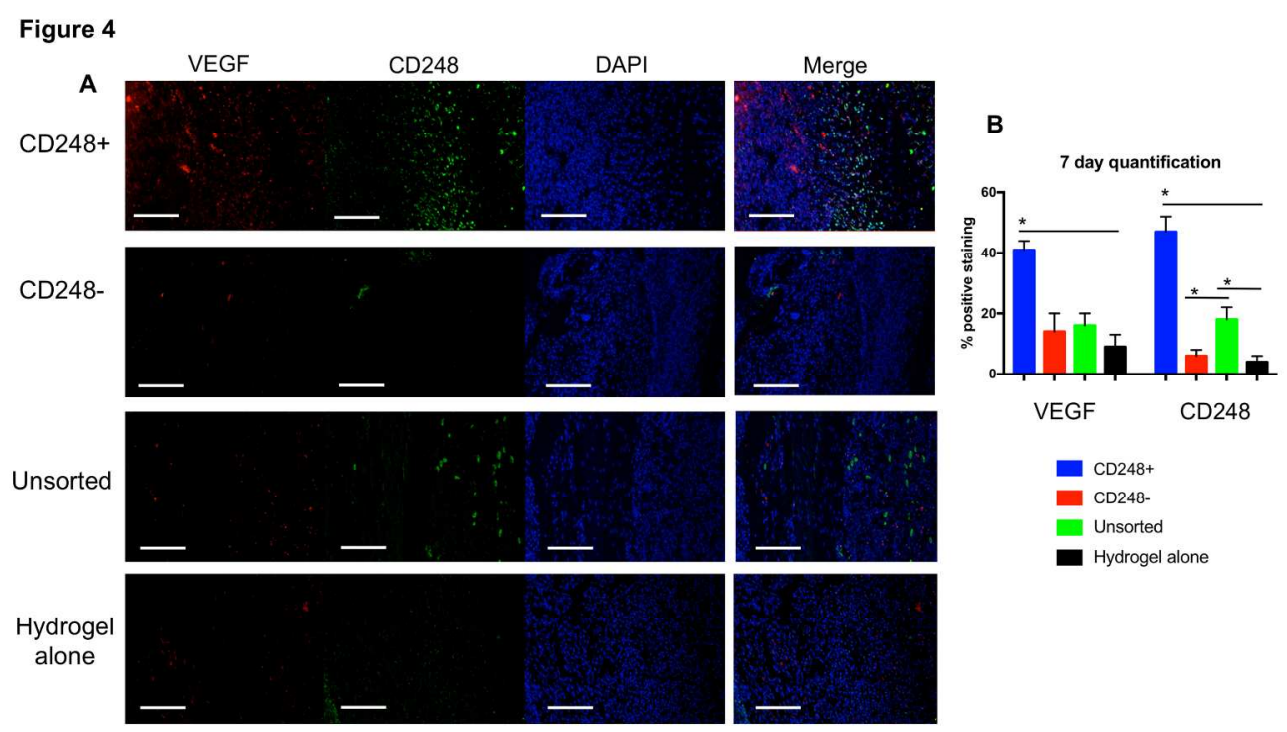

Figure 4: A) Immunohistochemical stains of wounds for VEGF (red), CD248 (green), and!! + DAPI (blue), with merged image on right. Scale bar $=100 \mu \mathrm{m}$. (B) Quantification of!! + immunofluorescent images (*p < $(1)$ $0.05)$. 


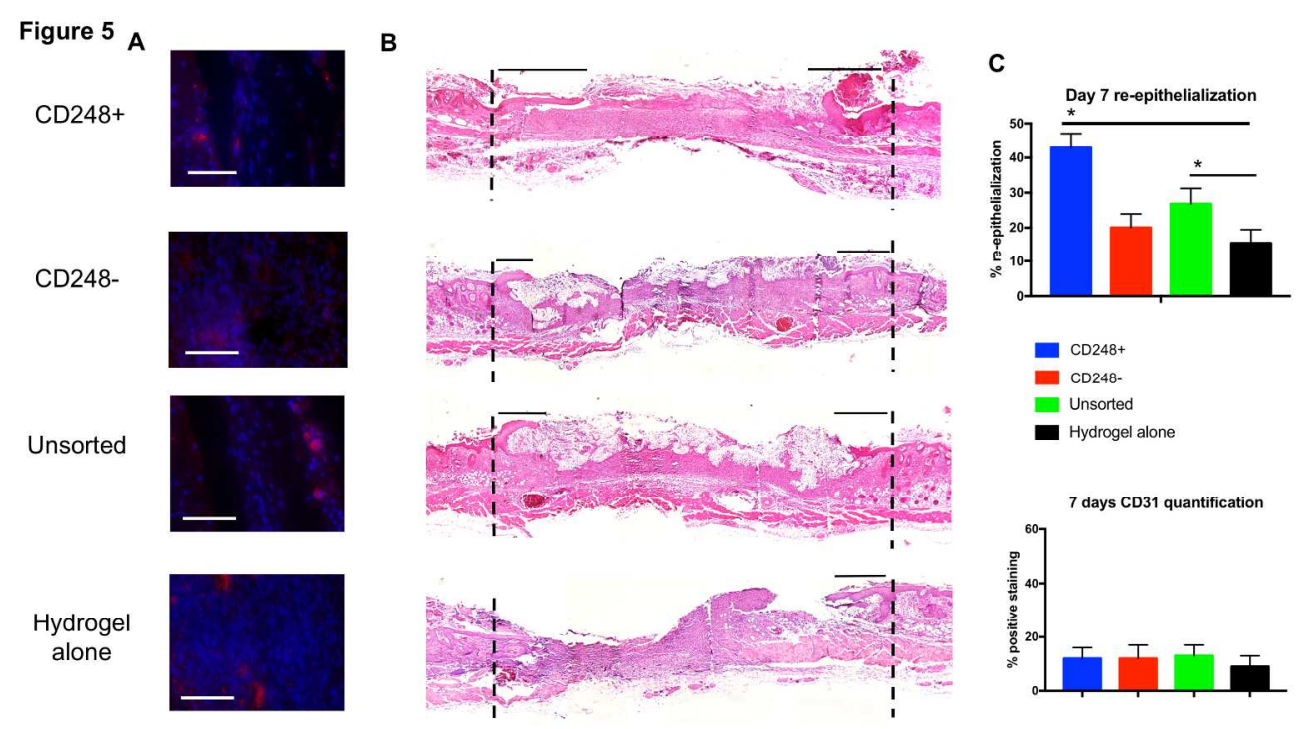

Figure 5: (A) CD31 immunohistochemical staining of wounds excised 7 days post-op. Scale!! + bar $=100$ $\mu \mathrm{m}$. (B) H\&E images of full wounds. Dotted lines represent wound borders, and!! + solid perpendicular lines highlight length of epithelial cover. Re-epithelialization is expressed!! + as a percentage of the distance between the dotted lines. (C) Quantification of reepithelialization!! + (top), and quantification of CD31

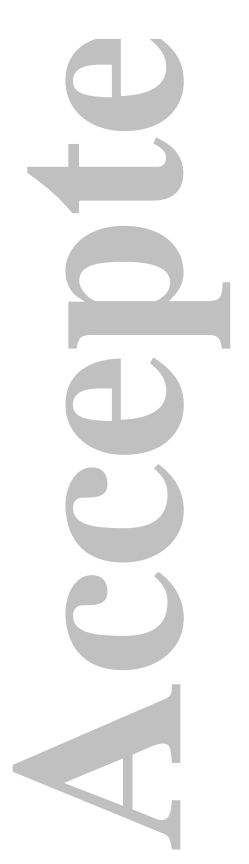
staining (bottom). $(* p<0.05)$. 


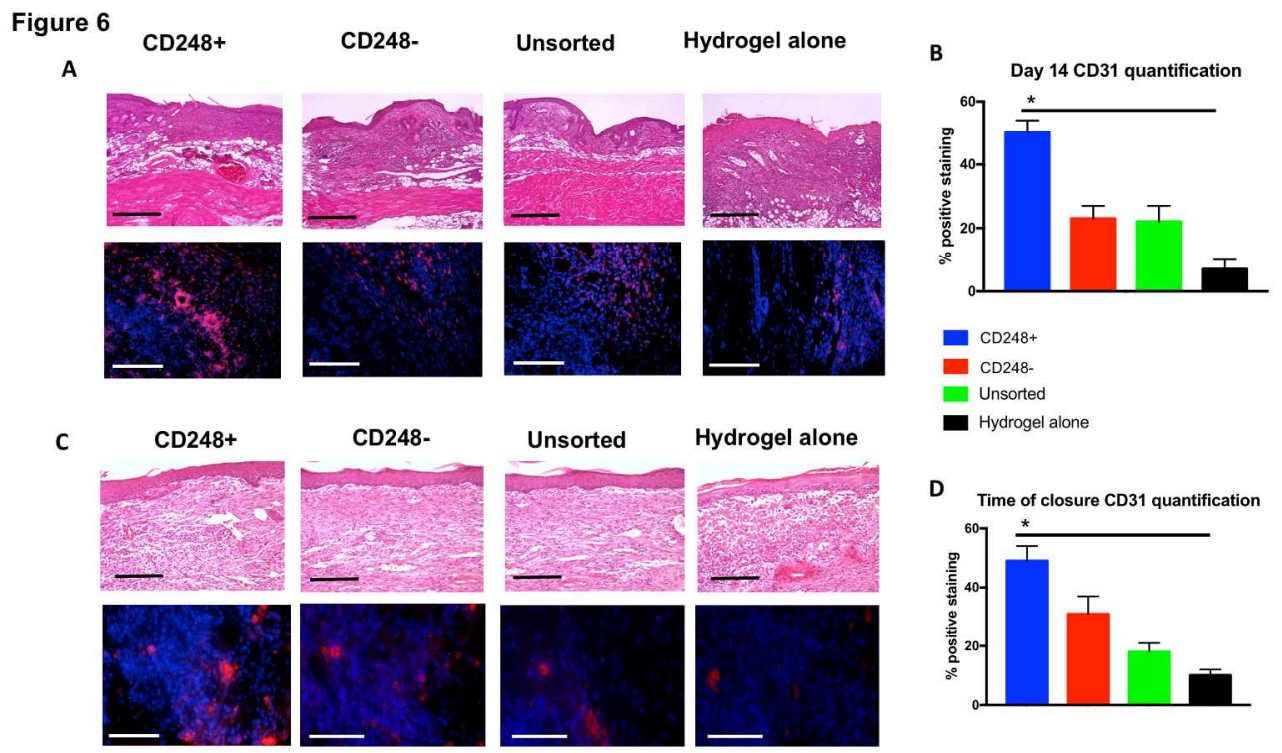

Figure 6: (A) Haematoxylin and eosin staining of excised wounds at day 14 (top row). Scale!! + bar $=80 \mu \mathrm{m}$. Anti-CD31 (red) immunofluorescent staining (bottom row) Scale bar $=100 \mu \mathrm{m}$.!! + (B) Quantification of CD31 immunofluorescent images shown in 6A revealed significantly! + more staining in CD248+ treated wounds compared to other groups $(* p<0.05)$. (C) H\&E!! + staining at completion of wound healing (top row). Scale bar $=80 \mu \mathrm{m}$. Anti-CD31 (red)!! + immunofluorescent staining (bottom row). Scale bar = $100 \mu \mathrm{m}$. (D) Quantification of CD31! + immunofluorescent images shown in 6C demonstrated more nascent vessels in the wounds!! + of CD248+ group when compared to the other 3 test groups. $(* p<0.05)$.

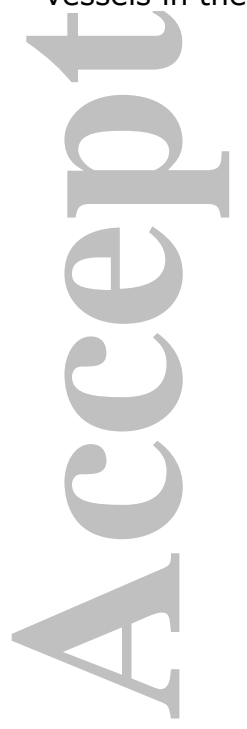




\section{University Library}

\section{- M M N E R VA A gateway to Melbourne's research publications}

Minerva Access is the Institutional Repository of The University of Melbourne

Author/s:

Brett, E;Zielins, ER;Chin, M;Januszyk, M;Blackshear, CP;Findlay, M;Momeni, A;Gurtner, GC;Longaker, MT;Wan, DC

Title:

Isolation of CD248-expressing stromal vascular fraction for targeted improvement of wound healing.

Date:

2017-05

Citation:

Brett, E., Zielins, E. R., Chin, M., Januszyk, M., Blackshear, C. P., Findlay, M., Momeni, A., Gurtner, G. C., Longaker, M. T. \& Wan, D. C. (2017). Isolation of CD248-expressing stromal vascular fraction for targeted improvement of wound healing.. Wound Repair Regen, 25 (3), pp.414-422. https://doi.org/10.1111/wrr.12542.

Persistent Link:

http://hdl.handle.net/11343/292844 